livraisons

d'Histoire

de l'Architecture

\section{Livraisons de l'histoire de l'architecture}

16 | 2008

Grands chantiers et matériaux

\title{
Le chantier de la fontaine des Quatre-Saisons, rue de Grenelle (1739-1745)
}

The construction of the Fontaine des Quatre-Saisons, rue de Grenelle, Paris

(1739-1745)

Der Bau des Brunnens der vier Jahreszeiten in der rue de Grenelle (1739-1745)

Dominique Massounie

\section{(2) OpenEdition}

Journals

Édition électronique

URL : http://journals.openedition.org/lha/183

DOI : $10.4000 /$ /ha. 183

ISSN : 1960-5994

Éditeur

Association Livraisons d'histoire de l'architecture - LHA

Édition imprimée

Date de publication : 10 décembre 2008

Pagination : 59-70

ISSN : 1627-4970

\section{Référence électronique}

Dominique Massounie, «Le chantier de la fontaine des Quatre-Saisons, rue de Grenelle (1739-1745)», Livraisons de l'histoire de l'architecture [En ligne], 16 | 2008, mis en ligne le 10 décembre 2010, consulté le 03 mai 2019. URL : http://journals.openedition.org//ha/183 ; DOI : 10.4000//ha.183

Ce document a été généré automatiquement le 3 mai 2019.

Tous droits réservés à l'Association LHA 


\section{Le chantier de la fontaine des Quatre-Saisons, rue de Grenelle (1739-1745)}

The construction of the Fontaine des Quatre-Saisons, rue de Grenelle, Paris (1739-1745)

Der Bau des Brunnens der vier Jahreszeiten in der rue de Grenelle (1739-1745)

\section{Dominique Massounie}

1 Entre 1739 et 1745, Edme Bouchardon, sculpteur du roi, travaille à la réalisation du décor de la nouvelle fontaine du faubourg Saint-Germain. D'ordinaire, c'est le maitre des bâtiments de la Ville qui fournit les plans, élévations et devis pour la construction des édifices hydrauliques: cette fois, il a dû se contenter de la rédaction du devis des ouvrages de maçonnerie à partir des dessins et devis des ouvrages de sculpture fournis par le sculpteur. En 1902, dans un article consacré à l'édifice, Alphonse Roserot expliquait le caractère exceptionnel de cette commande par l'amitié qui liait à l'artiste le commanditaire, le prévôt des marchands Michel-Étienne Turgot, et par l'attention que le cardinal de Fleury, premier ministre, auteur de l'inscription du frontispice, porta à l'érection de ce monument ${ }^{1}$. Ce chantier se signalait effectivement déjà par la qualité des acteurs, commanditaire et artiste, mais aussi par l'intérêt qu'il suscita, jusqu'aux plus hautes sphères du pouvoir. Le troisième traité de Vienne, signé le 18 novembre 1738, qui mettait fin à la guerre de succession de Pologne, inspira sans doute une modification du programme. L'événement est signalé sur la table de marbre noir qui surmonte la figure de la Ville ${ }^{2}$ :

Tandis que Louis XV, objet de l'amour de son peuple et excellent père, appui de la tranquillité publique, après avoir reculé sans répandre le sang les frontières $d u$ royaume de France et alors que la Paix vient d'être heureusement établies entre les Allemands et les Russes et les sujets de l'Empire Ottoman, régnait d'une manière à la fois glorieuse et pacifique, le prévôt des marchands et les édiles ont voué cette fontaine au service des citoyens et à l'embellissement de la ville, au cours de l'année $1739^{3}$. 
2 L'inscription en latin devait ne rien signifier pour bien des passants et seul le laurier, associé au chiffre royal de la frise d'entablement, évoquait la Paix. Pour le commun donc, il était bien difficile de comprendre qu'était apparu ici une nouvelle forme de monument triomphal, célébrant avec discrétion un triomphe clamé avec modestie. La seconde inscription, en français cette fois, portait à la postérité prévôt et échevins ainsi qu'Edme Bouchardon, "sculpteur du roi, né à Chaumont-Bassigny ». Les rondes-bosses de marbre blanc du frontispice, les Fleuves encadrant la figure couronnée de la Ville, formaient une allégorie du bon gouvernement de la cité.

\section{Nature du programme et postérité du monument}

3 La construction de la fontaine des Quatre-Saisons, rue de Grenelle, apparaît d'abord comme l'aboutissement des efforts renouvelés de la Ville pour équiper un quartier nouveau et prospère. Il n'est question durant de nombreuses années que de doter le faubourg Saint-Germain d'une fontaine publique semblable à beaucoup d'autres. Ordonnée en $1707^{4}, 1715^{5}$ et $1723^{6}$, sa construction ne fut mise en chantier qu'en 1739 lorsque la Ville apprit - sans que nous sachions quand et par qui - que les religieuses du monastère royal (je souligne) des Récollettes s'apprêtaient à faire clore leur terrain « d'un mur intérieur de 36 pieds de haut pour n'être point vues ». La Ville proposa alors de leur épargner «la dépense qui leur en coûterait, au moins une somme de 3000 livres $^{7}$ ", si elles consentaient à lui vendre la partie du terrain sur laquelle la fontaine serait construite, ainsi que le mur sur lequel elle s'appuierait. En effet, au lieu d'être adossée à une maison ou d'en occuper le rez-de-chaussée, cette fontaine s'appuie contre un mur de clôture, suffisamment haut pour dissimuler le jardin du couvent, mais percé d'une porte cochère pour pouvoir $\mathrm{y}$ accéder :

La fontaine [...] consiste dans un corps de bâtiment sur trois faces, dont la principale qui se présente sur la rue a 21 pieds de largeur $[6,80 \mathrm{~m}]$ et 37 d'élévation [12 m] divisée dans sa hauteur en deux parties. La première, qui sert comme de base à la seconde, est un grand socle d'architecture rustique, orné de refends dans toute son étendue. Elle s'élève à 12 pieds au-dessus du pavé de la rue et forme dans son milieu un corps avancé en tour ronde; au-dessus s'élève un second corps d'architecture décoré de colonnes et de pilastres d'ordre ionique qui portent leur entablement. Tout l'édifice est terminé par un fronton ${ }^{8}$.

Dans le premier devis, daté du 6 mars 1739, seul le corps central - la haute tour qui contient le réservoir - est considéré comme la fontaine adossée contre le mur rectiligne qui forme la clôture du couvent. Soubassement de la fontaine et mur sont ornés de lignes de refends qui créent néanmoins une impression d'unité entre les deux objets. La fontaine est à peine plus large que les deux ailes.

Le marché est passé le 6 mars, le terrain de soixante pieds de face acheté le 7. Dès le lendemain, Jean-Baptiste-Augustin Beausire lève le plan du terrain contigu, acquis le 13 mai suivant. Edme Bouchardon peut reprendre sa copie. Le marché de sculpture du 23 décembre porte sur un programme bien plus important: "Cette fontaine qui, suivant le premier dessin sur lequel le marché a été conclu, ne consistait que dans un seul corps de bâtiment de 21 pieds de face et 37 d'élévation, a présentement une étendue beaucoup plus considérable. L'on a ajouté deux ailes de bâtiment qui soutiennent la fontaine et lui donne un air de magnificence qu'elle n'avait point ${ }^{9}$. " La fontaine occupe désormais un terrain de 88 pieds de face (soit 28,51 m) et ne s'élève plus à 37, mais à 42 pieds $(13,61 \mathrm{~m})$ de haut. 
Le corps central s'avance au centre de cette nouvelle demi-lune décorée des Génies et des quatre bas-reliefs de Saisons (ill. 1).

III. 1 : Edme Bouchardon, fontaine des Quatre-Saisons, rue de Grenelle, Paris, 1739-1745

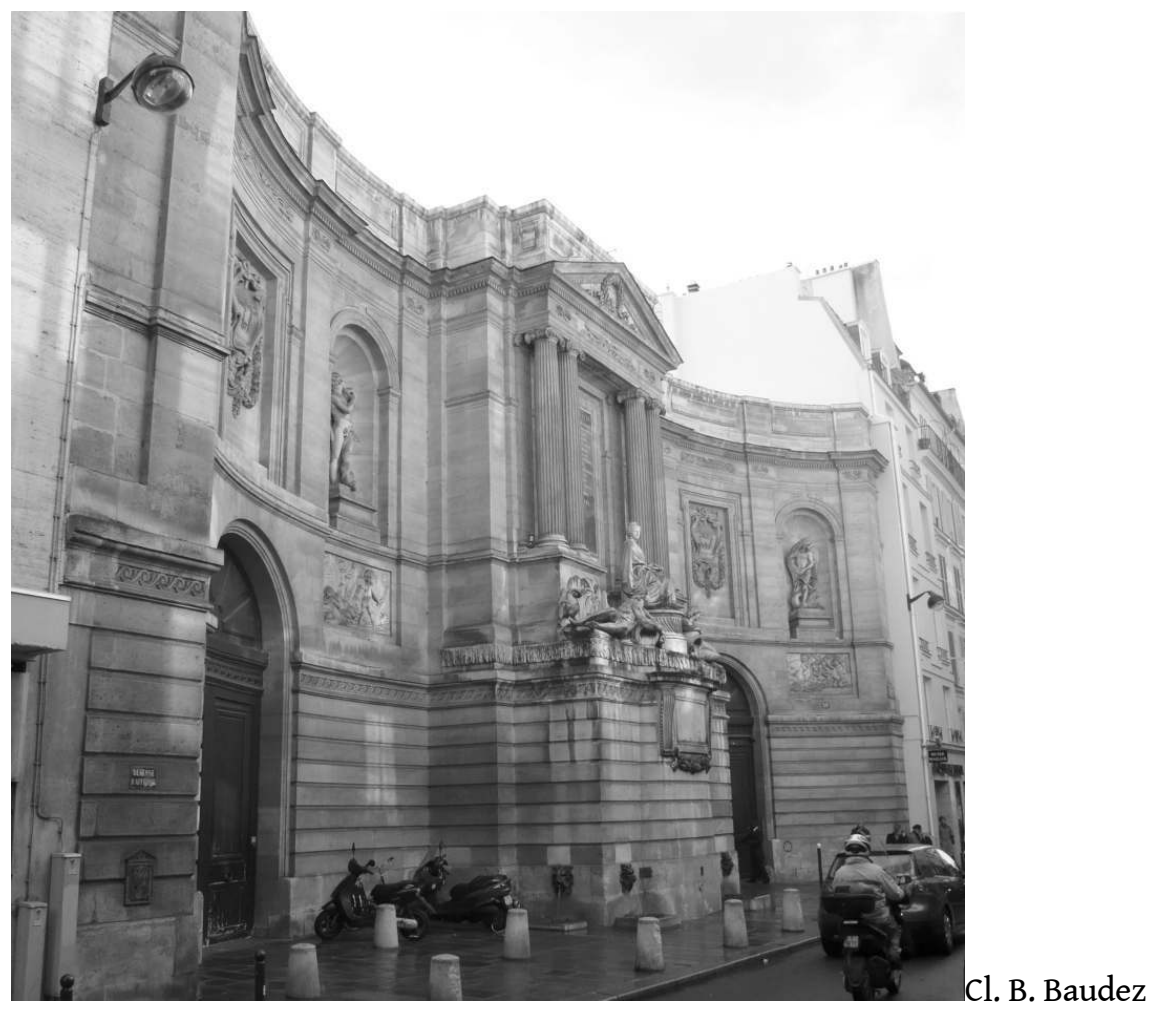

Le 11 février 1746, la Ville accordait à Edme Bouchardon une pension de 1500 livres, le prévôt et les échevins «ayant considéré que les ouvrages de sculpture en marbre et en pierre étaient si parfaitement achevés et d'une si grande beauté que ce monument élevé à la gloire de Sa Majesté ferait connaître, dans les temps les plus reculés, le goût de ce siècle et à quel point de perfection l'art de la sculpture a été porté par ledit sieur Bouchardon ; aucun ouvrage aussi digne de l'admiration générale, mériterait également de cette ville capitale une marque de reconnaissance envers le sieur Bouchardon, qui puisse en même temps exciter l'émulation de tous ceux qui s'adonnent aux arts et transmettre à la postérité un exemple des récompenses que méritent leurs talents et leurs [illisible] lorsqu'ils atteignent à un degré de perfection capable de faire honneur au goût et la magnificence de ce grand royaume ${ }^{10}{ }^{\prime}$. Alphonse Roserot en 1902 et Daniel Rabreau dans un article consacré à l'iconographie du monument publié en 1995 soulignaient que de célèbres amateurs, archéologues ou hommes de lettres, tels que Pierre-Jean Mariette, le comte de Caylus ou encore Voltaire, s'intéressèrent à l'édifice, avant, pendant et après sa construction $^{11}$. Malgré l'absence, maintes fois soulignée, d'eau, de jaillissements et de bassins, la fontaine de la rue de Grenelle s'imposa rapidement dans l'esprit des architectes et des amateurs comme le grand monument d'architecture de la capitale. En effet, quatre fontaines très différentes furent bâties à Paris après 1745: la fontaine du Diable, à l'angle des rues de l'Échelle et Saint-Louis, dont la construction débuta en 1753, la fontaine du Pot-de-Fer, rue Mouffetard, édifiée en 1758, et celle des Haudriettes, du dessin de Pierre-Louis Moreau, bâtie en 1766 à proximité de l'hôtel de Soubise. Ce sont des bâtiments ordinaires par leurs dimensions et la facture de leur décor. Le quatrième édifice, en revanche, bâti entre 1766 et 1770 et qui jouxte l'hôtel Saint-Florentin, à l'angle 
nord-est de la place Louis XV, en bordure des Tuileries, est du dessin d'Ange-Jacques Gabriel. Guillaume II Coustou le Jeune est l'auteur des Tritons qui ornent le tympan de son fronton. Cet édifice ne retiendra l'attention ni des théoriciens, ni des amateurs. Le chefd'œuvre de Bouchardon occupe les esprits, au XVIII ${ }^{\mathrm{e}}$ siècle et bien au-delà.

7 À deux reprises, Jacques-François Blondel choisit de décrire l'édifice pour expliquer le choix de l'ordre à employer pour les façades des monuments publics. En 1757, dans l'article «Fontaine » de l'Encyclopédie de Diderot et d'Alembert, il conteste le choix de l'ordre ionique pour un monument public soumis comme tout autre aux règles de la convenance et du caractère. Dans l'article "Architecture ", essentiellement consacré à l'usage des ordres, la fontaine de Grenelle sert à nouveau de contre-exemple ${ }^{12}$. Si l'ambition de la Ville était d'offrir aux architectes et amateurs l'occasion de débattre de l'idée du monument public autour d'un édifice digne d'une grande capitale, comparable par sa beauté à ceux que l'on peut observer à Rome, alors cette ambition, parfaitement légitime, était atteinte. Mais plus que ce frontispice ionique qui retint l'attention de Blondel, ce sont la statuaire et la composition du groupe central qui marquèrent les esprits (ill. 2).

III. 2 : Edme Bouchardon, corps central de la fontaine des Quatre-Saisons, rue de Grenelle, Paris, $1739-1745$

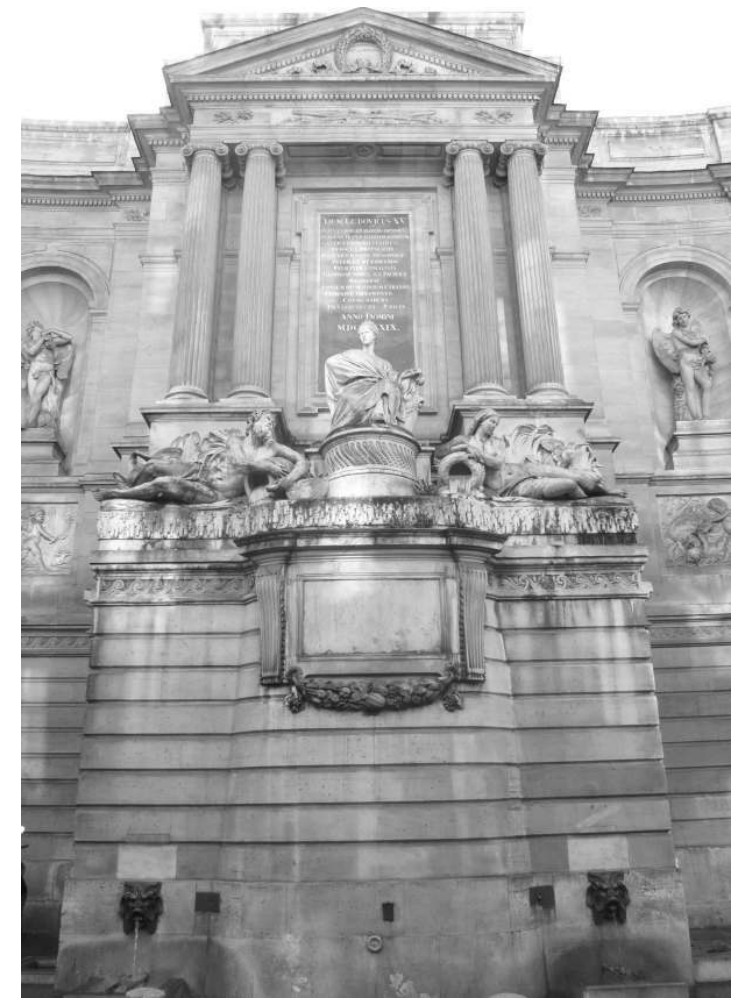

Cl. B. Baudez

La Ville et ses fleuves s'imposèrent comme un modèle pour les architectes comme pour les sculpteurs. La figure principale, selon les termes du devis du 6 mars 1739, « représente la ville de Paris, sous la figure d'une femme assise dans un trône placé sur un socle circulaire. Elle sera richement vêtue et l'on fera en sorte que son attitude inspire la majesté ; d'une main elle tiendra une proue de navire sur laquelle elle s'appuiera; elle aura l'autre bras replié et appuyé sur le côté et sur la tête, elle portera une couronne 
murale ${ }^{13}$ ». Figure allégorique semblable à la déesse Rome, elle emprunte également à Cybèle: la proue a remplacé le gouvernail, symbole de l'abondance des récoltes qui parvenaient à Rome par voie maritime, mais la couronne crénelée est bien celle de l'antique déesse de la fertilité et de l'abondance des biens de la terre, sous la protection de laquelle les villes aimaient à se placer. D'ailleurs le développement ultérieur du décor confirme cette interprétation: ayant supprimé les quatre gigantesques cornes d'abondance qui devaient encadrer les armes de la Ville, Bouchardon " a cru ne pouvoir rien imaginer de mieux que d'y placer dans des niches quatre figures debout [...] des Génies des saisons représentés ainsi qu'ils le sont sur les médailles par de jeunes gens ailés qui portent les fruits qui croissent dans chaque saison. [Il] a eu par là dessein de montrer que l'abondance qui règne dans Paris ne souffre aucune interruption durant le cours de l'année ${ }^{14}$ ». La rivière de Seine qui regarde la ville de Paris « avec une satisfaction mêlée de surprise $\mathrm{e}^{15}$ » et la rivière de Marne, " uniquement occupée du soin de répandre ses eaux bienfaisantes dans la ville, d'y procurer l'abondance et d'y faire fleurir le commerce ${ }^{16}$ " garantissent l'abondance des biens nourriciers que le fleuve fait entrer dans la capitale. La disposition des figures évoque la composition pyramidale réalisée par Michel-Ange pour la fontaine de l'escalier du palais des Sénateurs, à Rome, place du Capitole, mais l'unité de ce groupe et la majesté de la figure centrale à laquelle la nature se subordonne sont inédits.

9 Si l'absence d'eau dans les conduites de la capitale rend inopérante la critique concernant les jaillissements et les bassins, les circonstances de la construction, le choix de la rue et les dimensions du terrain, comme du monument, rendent le débat du dégagement de ce monument également parfaitement stérile. Néanmoins ce débat témoigne également des qualités reconnues à cet édifice. À l'instar de Voltaire, Marc-Antoine Laugier, JacquesFrançois Blondel puis Maille Dussausoy réclament la création d'une place au-devant de la fontaine. «Il faut que les fontaines soient élevées dans les places publiques et que les beaux monuments soient vus de toutes les portes ${ }^{17}$ ", s'écriait Voltaire dès 1739. Et Laugier de désirer, en 1753, " une place plus commode et plus avantageuse ${ }^{18}$ ", Blondel, en 1762, " un tout autre point de vue ${ }^{19}$ ", et Dussausoy, cinq ans plus tard, " une très belle place $^{20}$ » pour le chef-d'œuvre de Bouchardon. Seule la beauté du monument peut justifier sous la plume de ces hommes le retournement inédit de la formule qui consiste d'ordinaire à proposer la construction d'une ou plusieurs fontaines pour l'embellissement d'une place. Cette fois, ce n'est plus la place qu'il faut orner, mais la fontaine qui leur paraît réclamer une place. Il faut attendre la construction de la fontaine de Trevi à Rome pour que l'idée de proportionner l'espace à l'observation d'un monument soit renouvelée par les architectes.

\section{Nature des ouvrages et comptes du chantier}

L'histoire du chantier permet de comprendre comment la Ville et l'artiste parvinrent ensemble à la fabrique du monument. Le choix d'un sculpteur exceptionnel, celui d'un emplacement privilégié, un budget considérable et un ensemble de précautions propres à garantir la qualité de la construction font déjà de l'édifice un objet hors du commun. Trois devis sont établis pour l'érection et la décoration de la fontaine. Deux d'entre eux portent sur les ouvrages de sculpture "tant en marbre qu'en pierre et en bronze ». Le 6 mars 1739, un premier marché est passé avec Edme Bouchardon pour la somme de 49518 livres ${ }^{21}$. Selon le devis qui commence par une description précise des ouvrages d'architecture et 
de sculpture figurés sur le dessin, les sommes qui accompagnent chacune des rubriques de «l'état de la dépense pour les sculptures » correspondent à l'exécution des ouvrages, depuis le dessin jusqu'à la finition de chacun des objets. Il n'est jamais question du coût des matériaux. Ainsi, la réalisation du modèle et l'exécution en marbre de la grande figure de la Ville sont payées 12000 livres au sculpteur, celles des deux figures couchées qui l'accompagnent 20000 livres, celles du piédestal de la Ville 150 livres, celles des deux mascarons de bronze 800 livres, etc. En outre, le devis contient plusieurs rubriques qui portent uniquement sur le travail de préparation du sculpteur :

Comme il conviendra, en attendant l'exécution des marbres, de modeler dans la grandeur de l'ouvrage les trois figures de la ville de Paris, de la Seine et de la Marne et qu'il faudra pour y parvenir modeler aussi en grand tout le corps d'architecture qui leur sert de base, qu'il faudra ensuite faire mouler les dites figures et les jeter en plâtre, l'on ne croit pas pouvoir estimer cette augmentation de travail [je souligne] qui est très considérable moins de 5000 livres $^{22}$.

La rubrique "Observations", qui clôt le devis, contient la description des ouvrages demeurant à la charge de la Ville : achat des pierres et leur transport dans l'atelier du sculpteur, puis jusqu'à la fontaine ; construction des échafaudages nécessaires à la pose des figures et leur scellement; conduite des travaux de construction; exécution d'un petit modèle en bois de l'édifice si nécessaire. Les 50000 livres, ou peu s'en faut, tout comme les 32667 livres du second marché passé le 23 décembre $1739^{23}$ pour les ouvrages entrant " dans le nouveau dessein ", soit quatre figures et quatre bas-reliefs en pierre de Tonnerre figurant les Saisons, et quelques ornements de plus, sont le prix de cinq années de travail d'un artiste réputé et de son atelier. Dans les devis de sculpture rédigés par le sculpteur, on apprend également que son nouveau dessin, «les épures qu'il a tracées en grand sur la muraille du couvent des Jacobins ", les grandes planches séparées qu'il a sans doute exécutées pour l'entrepreneur et « tous les soins qu'il s'est donné depuis près d'une année [...] pour mettre les ouvriers en état de construire le bâtiment » lui seront payés 3000 livres $^{24}$. Le premier devis portant sur l'iconographie, la nature et la taille des sculptures ne contient que des informations ordinaires sur les matériaux: il n'est question que de marbre blanc et de pierre dure sans mention de provenance, ainsi que de bronze. Dans le second devis, il est fait mention de la pierre de Tonnerre, pour les Génies et les bas-reliefs des Saisons, et d'un contrat avec Louis Trouard, marbrier de la Ville, pour le sciage des marbres. Le premier devis contient en outre un calendrier de chantier et un échéancier de paiement: tandis que Bouchardon s'oblige à achever son ouvrage pour le 15 août 1740, à l'exception des grandes figures de marbre qu'il doit livrer dans les quatre ans, la Ville s'engage à lui verser le quart de la somme totale à la signature, un quart au mois de septembre suivant, un troisième quart à la réception des premiers ouvrages le 15 août 1740 , et le dernier, au terme des travaux.

Quelques quittances et mémoires permettent de se rendre compte de ce que l'exécution de rondes-bosses en marbre ajoute à une dépense rendue déjà exceptionnelle par le coût du travail. Les marbres blancs utilisés pour les trois grandes figures du corps central proviennent des magasins du roi. Un premier bloc de marbre blanc est acheté le 25 novembre 1738 pour la somme de 4240 livres et doit être « employé à l'une des statues représentées dans le dessin de la fontaine projetée rue de Grenelle ${ }^{25}$ ». Les deux « blocs de marbre blanc contenant 126 pieds, 10 pouces, 6 lignes cubes » achetés le 28 janvier 1739, " pour être employés [...] suivant le dessein qui sera par nous [les prévôt et échevins] arrêté ", coûtent à la Ville 5073 livres et 10 sols $^{26} ; 200$ livres sont nécessaires à leur transport dans la cour du Louvre. Le quatrième marbre de 70 pieds cubes, acheté au sieur 
Coustou le 5 avril 1740 pour la somme de 2100 livres $^{27}$, correspond sans doute au piédestal circulaire de la figure centrale. La dépense pour le marbre blanc statuaire s'élève donc à 11413 livres. Le même mémoire consacré à la fontaine fait état d'un paiement de 2600 livres à Étienne Piot, voiturier, pour six blocs de pierre de Tonnerre livrés dans l'atelier de Bouchardon, dans la cour du vieux Louvre ${ }^{28}$. Les dépenses consenties par la Ville pour l'achat des pierres et leur transport s'élèvent donc au moins à 18393 livres, auxquelles il faut sans doute ajouter les frais de transport du premier et du quatrième marbre et peut-être le prix de deux blocs de pierre de Tonnerre supplémentaires si l'on considère que les Génies des saisons sont au nombre de quatre, tout comme les bas-reliefs qu'ils surmontent. Nous n'avons pas non plus connaissance du prix des deux tables de marbre qui portent des inscriptions. Raisonnablement, on peut estimer l'achat des matériaux destinés au décor à près de 20000 livres. C'est donc un peu plus de 100000 livres que la Ville consacra au décor de ce monument. Le 17 décembre 1745, le maître des bâtiments de la Ville effectuait la réception des ouvrages de sculpture 29.

13 Le 10 juillet 1739, la Ville passe marché avec l'entrepreneur Jacques Megrelin, demeurant rue de la Mortellerie, paroisse Saint-Paul, sur la base du devis établi par Jean-BaptisteAugustin Beausire $^{30}$. La maçonnerie doit être achevée en juin 1740. Les matériaux « de qualité » - provenant de la démolition de deux maisons acquises par la Ville et de l'ancienne clôture du couvent des Récollettes - seront employés pour les fondations, ainsi que les bois pour construire une « forte cloison de charpente » destinée à fermer la bassecour des religieuses pendant le temps de la construction. Le mortier mêle sable de rivière et chaux de Melun et de Senlis. Moellons et libages des fondations et du puisard seront de pierre dure d'Arcueil, tandis que les murs de face et voûtes des mêmes constructions souterraines seront formés de pierres de taille des carrières d'Arcueil et fonds de Bagneux, tout comme les murs en élévation jusqu'à douze pieds de hauteur, soit la hauteur du soubassement orné de lignes de refends, jusqu'au-dessous de la frise de postes qui le couronne. Au-dessus sera employée la pierre tendre «la plus dure et la plus parfaite » des carrières de Conflans-Saint-Honorine, toutes les assises « posés sur calles de plomb et mortier de sable de rivière et chaux ${ }^{31} »$. La double nature du monument - un corps central servant de fontaine et deux ailes qui sont en réalité des murs de grande hauteur - justifie sans doute la hauteur de ce soubassement de pierre dure qui n'est habituellement qu'une plinthe formée par les trois premières assises. La pierre dure d'Arcueil est de nouveau utilisée pour les éléments de couronnement de l'édifice, les dalles et voûtes intérieures, les escaliers, les bornes et bancs disposés au-devant de la fontaine. Les quatre colonnes de l'avant-corps sont de pierre de Conflans, d'une seule pièce chacune épannelée à la carrière. Enfin, les deux rampants du fronton sont formés d'une seule grande pierre chacun «de liais Cliquart le plus franc ${ }^{32}$ ». Les différentes pierres employées pour la fontaine de Grenelle sont non seulement des pierres d'une grande solidité, mais également d'une belle qualité de grain. On ne peut pas dire pour autant que cet édifice se distingue des autres fontaines dont la maçonnerie est toujours d'excellente qualité, étant donné le danger que représente l'eau pour toute construction.

Le devis est suivi du marché à la toise, contenant les prix fixés à la toise superficielle ou à la toise carrée pour chaque nature d'ouvrage, puis du marché passé avec l'entrepreneur qui sera payé « au fur et à mesure de l'avancement des ouvrages suivant les toisés qui en seront faits par le maître général des bâtiments de la Ville». Cette procédure, qui remplace l'adjudication au rabais, est tout à fait exceptionnelle : Jean Beausire ${ }^{33}$, le père 
de Jean-Baptiste-Augustin, qui assiste encore son fils à cette date, n'a pas pour habitude de recourir au marché à la toise. Particulièrement attentif au contrôle des dépenses, il lui arrive même parfois, après la réception des travaux, de soustraire quelques livres à la somme arrêtée après adjudication au rabais, pour différence de façon ou de dimensions. Le marché à la toise induit un contrôle régulier des travaux pour établir des toisés intermédiaires; ainsi, la qualité de la mise en œuvre est-elle plusieurs fois vérifiée. D’ailleurs, le 10 juin 1739, la Ville désigne un inspecteur des travaux, Jean Pallu de Fessert, dont les appointements annuels s'élèvent à 800 livres $^{34}$. À supposer qu'il ait exercé ses fonctions jusqu'à réception des travaux, il aurait perçu 4800 livres, somme qui correspond au prix d'une fontaine ordinaire. Le soin que l'on veut apporter à la construction du monument est manifeste. Malheureusement pour l'historien, le marché à la toise ne permet pas de savoir directement combien la Ville dut débourser pour la construction. En l'absence de toisé et de quittances de paiement ou de mémoire récapitulatif portant sur ces ouvrages, il est très difficile d'évaluer la dépense: les dimensions de l'édifice - 42 pieds de hauteur $(13,61 \mathrm{~m})$ sur 88 pieds de largeur $(28,51 \mathrm{~m})$ laissent supposer que quelques dizaines de milliers de livres ont été nécessaires. Encore une fois, la fontaine des Quatre-Saisons de la rue de Grenelle apparaît par son coût, le second du XVIII siècle après celui de la place Louis XV, comme l'un des plus gros chantier jamais entrepris par la Ville, à tel point que toute tentative de comparaison avec la dépense ordinairement consentie pour la construction d'une fontaine est bien peu signifiante - la fontaine Saint-Avoye, édifiée entre octobre 1742 et juillet 1743, a coûté 3802 livres, 6 sols, 6 deniers ${ }^{35}$.

15 Un dernier élément ajoute au caractère exceptionnel de ce chantier. Alors que la Ville a pour habitude de construire ses fontaines sur des terrains que les propriétaires, particuliers ou communautés religieuses, lui cèdent contre un filet d'eau, la construction de cette fontaine nécessite l'achat de trois parcelles importantes. La première, de 34 toises et demie, 17 pieds et 6 pouces superficiels, estimée 160 livres la toise, soit 5597 livres, 15 sols et 6 deniers, et dont le plan a été établi cinq jours auparavant par JeanBaptiste-Augustin Beausire, est achetée le 7 mars 1739, au lendemain de la signature du premier marché de sculpture, aux révérendes dames abbesse et religieuses du monastère royal des Récollettes de l'Immaculée Conception de la Vierge ${ }^{36}$. Le jour suivant, Beausire lève le plan de la maison attenante que la Ville achète le 13 mai pour la somme de 13000 livres $^{37}$ ! Enfin, le $1^{\text {er }}$ juillet 1739 , la Ville acquiert la maison de la veuve Pelletier, contiguë à la précédente, pour 14500 livres $^{38}$ ! L'achat des trois parcelles a donc coûté un peu plus de 33000 livres, mais l'acquisition du second et du troisième terrain a permis l'agrandissement du monument dans des proportions considérables.

Hors coût de construction, nous pouvons déjà estimer la dépense à quelques 136000 livres - 83000 livres pour la sculpture, 20000 livres pour les pierres utilisées uniquement pour le décor, 33000 livres pour le terrain - et affirmer sans risque qu'elle a dépassé les 150000 livres, soit le prix d'un petit hôtel particulier. En 1739, année de la commande, la Ville consacrait 100000 livres à l'achat de riz pour le soulagement des pauvres ${ }^{39}$, construisait le réservoir du Grand Égout qui reçut les 12, 14 et 16 juillet 1740 la visite du roi, de la reine et même celle du dauphin ${ }^{40}$ et célébrait somptueusement le mariage d'Élisabeth, fille de Louis XV, avec l'infant Philippe d'Espagne. L'heure n'était pas à l'économie. Une telle dépense pour la fontaine du faubourg Saint-Germain traduisait nécessairement des ambitions très grandes. Donner à l'édifice le statut d'un monument 
public, au sens moderne du terme, monument commémoratif et triomphal, au roi, à la paix et à la Ville, était sans doute à ce prix.

\section{NOTES}

1. 1. Alphonse Roserot, «La fontaine de la rue de Grenelle à Paris par Edme Bouchardon (1739-1745) », Gazette des Beaux-Arts, novembre 1902, p. 361-362.

2. 2. Daniel Rabreau, «Fontaine des Quatre-Saisons ou de Grenelle », Paris et ses fontaines de la Renaissance à nos jours, Dominique Massounie, Pauline Prévost-Marcilhacy, Daniel Rabreau dir., Paris, Action artistique de la Ville de Paris, 1995, p. 100.

3. 3. Traduction de Jean Bonfin, citée par Daniel Rabreau, ibid.

4. 4. Arch. nat., E 1939, arrêt du 23 août 1707.

5. 5. Arch. nat., E 1979, fo 243 et 244, arrêt du 2 mars 1715. La Ville doit faire l'acquisition d'une " place en chantier formant encoignure, occupée par un marbrier ", au carrefour des rues du Bac et de l'université. Sur le plan annexé à l'arrêt apparaît un édifice rectangulaire (fig. 235), doté de la cuvette de distribution nécessaire aux différentes concessions qui seront consenties aux propriétaires des hôtels environnants. Un pan coupé permet de dégager un espace de service protégé par quatre bornes disposées en arc de cercle et de décorer l'édifice d'une niche concave encadrée de deux piédroits et de deux colonnes engagées.

6. 6. Arch. nat., E 2043, f ${ }^{\circ} 451 \mathrm{v}-453 \mathrm{v}$, arrêt du 19 juin 1723. Il s'agit d'un nouvel emplacement, côté sud de la rue de Bourbon, entre les rues du Bac et de Poitiers.

7. 7. Arch. nat., MC, étude C, liasse 552 : contrat passé devant Marchand, notaire, pour la vente du terrain, le 7 mars 1739.

8. 8. Arch. nat., $\mathrm{H}^{2} 2023$, devis du 6 mars 1739.

9. 9. Ibid.

10. 10. Arch. nat., K 1058.

11. 11. Alphonse Roserot, « La fontaine de la rue de Grenelle à Paris par Edme Bouchardon (1739-1745) », op. cit., p. 353-372; Daniel Rabreau, «Fontaine des Quatre-Saisons ou de Grenelle », op. cit., p. 98-103.

12. 12. Encyclopédie ou Dictionnaire raisonné des sciences, des arts et des métiers, Paris, 1762, t. XVIII, p. 7 et pl. XIII.

13. 13. Arch. nat., $\mathrm{H}^{2} 2023$, devis du 6 mars 1739.

14. 14. Ibid., devis du 23 décembre 1739.

15. 15. Ibid., devis du 6 mars 1739.

16. 16. Ibid.

17. 17. Lettre de Voltaire au comte de Caylus citée par Alphonse Roserot, « La fontaine de la rue de Grenelle à Paris par Edme Bouchardon (1739-1745) », op. cit., p. 370, n. 454.

18. 18. Marc-Antoine Laugier, Essai sur l'architecture, Paris, Duchesne, 1753, p. 166. 
19. 19. Encyclopédie, op. cit., t. XVIII, commentaire de la planche XIII, p. 7.

20. 20. Maille Dussausoy, Le Citoyen désintéressé, ou diverses idées patriotiques concernant quelques établissements et embellissements utiles à la ville de Paris, Paris, Gueffier, 1767, t. I, p. 11 : «Par trop de précipitation et parce sans doute nous négligeons de voir les choses dans le grand, d'en considérer les suites et de faire un plan général, non seulement de l'édifice projeté, mais de tous ses accessoires, nous nous trouvons obligés de détruire en même temps que nous élevons et souvent de laisser des ouvrages imparfaits ; de là tant d'édifices superbes, isolés et comme perdus, qui auraient pu produire le plus grand effet et qui se trouvent déplacés ; tels sont le superbe portail de Saint-Gervais, [...] celui de Saint-Sulpice [...] ; telle est la belle fontaine de la rue de Grenelle, que le célèbre Bouchardon n'entreprit que dans l'idée qu'elle serait accompagnée d'une très belle place, qui en effet avait été projetée par M. Turgot, alors prévôt des marchands. »

21. 21. Arch. nat., $\mathrm{H}^{2} 2023$, devis de sculpture du 6 mars 1739.

22. 22. Ibid.

23. 23. Arch. nat., $\mathrm{H}^{2} 2023$, devis de sculpture du 23 décembre 1739.

24. 24. Ibid.

25. 25. Arch. nat., $\mathrm{F}^{14} 187^{\mathrm{A}}$, mémoire «Fontaine de Grenelle »; cette information est annotée « Min. R 81 fol. 45 »; voir Registre des délibérations du corps de Ville, $\mathrm{H}^{2 *} 1858$ (du 16 août 1738 au 15 août 1740).

26. 26. Ibid. ou $\mathrm{H}^{2} 1939^{1}$.

27. 27. Ibid.

28. 28. Arch. nat., $\mathrm{F}^{14} 187^{\mathrm{A}}$.

29. 29. Ibid.

30. 30. Arch. nat., $\mathrm{H}^{2}$ 2022, devis de maçonnerie et marché, 2 juin 1739.

31. 31. Ibid.

32. 32. Ibid.

33. 33. Isabelle Dérens, "Un siècle d'édiles parisiens : Jean Beausire et sa lignée ", Paris et ses fontaines de la Renaissance à nos jours, Dominique Massounie, Pauline Prévost-Marcilhacy, Daniel Rabreau dir., Paris, Action artistique de la Ville de Paris, 1995, p. 132-142.

34. 34. Arch. nat., $\mathrm{F}^{14} 187^{\mathrm{A}}$ ou $\mathrm{H}^{2} 1939^{1}$.

35. 35. Arch. nat., $\mathrm{H}^{2}$ 2023, réception des travaux, 2 août 1743.

36. 36. Arch. nat., MC, étude C, liasse 552, 7 mars 1739.

37. 37. Arch. nat., MC, étude C, liasse 553, 13 mai 1739.

38. 38. Ibid., $1^{\text {er }}$ juillet 1739 .

39. 39. Arch. nat., $\mathrm{H}^{2 *} 1858$, délibération du 6 mai 1739.

40. 40. Ibid., relations des cérémonies. 


\section{RÉSUMÉS}

L'histoire de la fontaine des Quatre-Saisons est sans conteste l'épisode le plus prestigieux de la carrière du sculpteur qui en réalisa le décor, Edme Bourchardon, qualifié par la Ville, le commanditaire de ce monument royal d'un nouveau genre, de Phidias moderne. L'exceptionnelle qualité des rondes-bosses du corps central et des niches latérales ainsi que des bas-reliefs a relégué au second plan l'étude de la construction de l'édifice dont les dimensions et le coût le classent également parmi les exceptions. Les archives du bureau de la Ville et les archives notariales nous permettent de comprendre dans quelles circonstances il a vu le jour. Elles nous renseignent sur l'achat des terrains, celui des marbres statuaires et des pierres de Tonnerre, sur les émoluments de l'artiste, la nature des ouvrages de maçonnerie, le prix des mascarons de bronze et la rémunération de l'inspecteur des travaux. Si la qualité des ouvrages est parfaitement connue, le coût global de la construction ne peut qu'être l'objet d'une estimation. En effet, le marché passé le 3 juin 1739 avec Jacques Mégrelin, sur la base du devis rédigé par Jean-BaptisteAugustin Beausire, comporte uniquement le prix de la toise fixé pour chacun des ouvrages. En l'absence du toisé, est-il possible d'évaluer la dépense? Elle a été considérable puisque ce chantier s'élevait déjà, hors paiements des ouvrages de maçonnerie et de plomberie, à plus de 139000 livres. Elle fait de ce monument public, à la fois fontaine et monument commémoratif, vraisemblablement l'édifice le plus coûteux jamais construit pour l'embellissement des villes à l'époque moderne.

The story of the Fontaine des Quatre-Saisons is indisputably the most prestigious episode in the career of the decoration's sculptor, Edme Bouchardon, an artist called the modern Phidias by his client, the City of Paris. The exceptional quality of the sculpture, statues in the round and low relieves, overshadowed the construction itself, which was also exceptional in cost and dimensions. We can retrace its history by analysing archives of the City Committee and of the Parisian notaries. We learn how the City bought the land, the marbles and the building stones extracted in the Tonnerre area; we are informed about the artist's remuneration, about the nature of the masonry, the price of the bronze grotesque masks and the works' surveyor's salary. The quality of each of the works is well known but the total cost of the construction can only be estimated. Indeed, the contract stroke the $3^{\text {rd }}$ of June 1739 with Jacques Mégrelin based on the quotation written by Jean-Baptiste-Augustin Beausire shows only the price of the toise for each of the work to be done. Without the measurement, can we estimate the expense? The latter was nevertheless considerable, because the construction's budget climbed already, without payments for masonry and plumbing, up to 139000 livres. Therefore, we can say that this public monument, a fountain and a commemorative monument altogether, is certainly the most costly edifice ever built for urban beautifying in the modern era.

Die Geschichte des Pariser Brunnens der vier Jahreszeiten ist zweifellos die prächtigste Episode der Laufbahn des Bildhauers Edme Bouchardon, der das Dekor schuf, insofern dass die Stadt, die Auftraggeber dieses königlichen Gebäudes neuer Art war, Bouchardon den modernen Phidias nannte. Im Vergleich zu der außerordentlichen Qualität der Vollplastiken des Hauptgebäudes und der Nebennischen sowie der Flachreliefs wurde die Baugeschichte lange als zweitrangige Studie angesehen, obwohl das Gebäude der Größe und den Baukosten nach ebenso einzigartig ist. 
So werden in Stadtarchiven und Notarakten alle möglichen Informationen erteilt, nicht nur über Bauumstände und verschiedene Maurerarbeiten, über Ankauf des Grundstücks und Preise des Marmors, der Steine aus Tonnerre oder der großen Bronzemasken, sondern auch über die Gehälter des Künstlers und des Bauinspektors. Alles zeugt zwar von der besonderen Qualität des Baus, aber dessen Gesamtkosten können nur geschätzt werden. In dem am 3. Juni 1739 mit Jacques Mégrelin abgeschlossenen Bauvertrag, gestützt auf dem Voranschlag von Jean-BaptisteAugustin Beausire, wird allerdings der Preis für jede Bauarbeit pro Toise (damaliges Längenmaß) bestimmt. Wie sind aber ohne dieses Maß heute die genauen Kosten einzuschätzen? Es handelt sich jedenfalls um eine beträchtliche Summe, die sich schon vor der Bezahlung der Maurer-und Bleigießarbeiten auf 139.000 Pfund bezog. So kann dieses öffentliche Gebäude, zugleich Quelle und Denkmal, wahrscheinlich für den kostspieligsten Bau gehalten werden, der je in der modernen Zeit "zur Stadtverschönerung erstellt wurde.

\section{AUTEUR}

\section{DOMINIQUE MASSOUNIE}

Dominique Massounie, née en 1971, est maître de conférences en histoire de l'art moderne à l'université Paris X-Nanterre. Elle a soutenu en décembre 2000, à l'université Paris I et sous la direction de Daniel Rabreau, une thèse de doctorat consacrée aux Monuments hydrauliques urbains : aqueducs, châteaux d'eau et fontaines dans la ville moderne, 1661-1791 (Monum éditions, collection Temps et espace des arts, à paraître). Ses recherches l'ont conduite à participer à plusieurs reprises à des colloques et publications consacrés à l'histoire des techniques. Sa maîtrise d'histoire, entreprise sous la direction de Daniel Roche, a été publiée en 1998 chez Nathan, dans la collection Jeunes Talents, sous le titre L'architecture des écuries royales du château de Versailles. Elle a participé, sous la direction de Jean-Michel Leniaud, à la publication des Procèsverbaux de l'Académie des beaux-arts au XIX ${ }^{\mathrm{e}}$ siècle. Depuis 2004, elle se consacre à l'étude du livre d'architecture au XVIII ${ }^{e}$ siècle et a organisé plusieurs rencontres autour de ce thème. Adresse électronique : massounie@aol.com. 